\title{
Can digital games serve as potential intervention or suicide risk?
}

\author{
Elsie Ong ${ }^{1}$ \\ ${ }^{1}$ Faculty of Education, University of Hong Kong, \\ elsieong@hku.hk
}

\begin{abstract}
With the rapid growth of advanced digital technology, the use of mobile apps is of increasing interest as a means for providing continuous access to evidence-based digital interventions. Suicide is a socially taboo topic with approximately 800,000 people dying due to suicide every year. As suicidal ideation and suicide risk increase rapidly, gamified intervention is argued to have an important role in the future of mental health care provision and access to these mobile resources may save lives. However, due to the lack of published research to demonstrate that apps can be effective at reducing suicide behaviours, many developers are pushing ahead with apps for suicide prevention with unclear benefits and risks. In this communication piece, issues and challenges of digital interventions will be discussed, hence solution such as using attentional bias modification may be an option for future health care. Overall, the use of digitized gamified intervention holds great promise for a radically new approach to deliver prevention programs, thus overcoming barriers inherent in traditional therapeutic approaches.
\end{abstract}

Keywords: Gamified intervention, suicide, mobile apps, cognitive deficits;

\section{Introduction}

Suicide is a socially taboo topic, yet approximately 800,000 people die due to suicide every year, which is one person every 40 seconds [1]. It is the tenth leading cause of death for all ages [2], but the incidence is even greater for people aged 15-to-24 years old where it is second largest cause. The issue of suicide is even more alarming when individuals do not tend to present to services when feeling suicidal [3, 4]. Although there has been a wealth of research in the study of suicidal behaviour, prediction and prevention of suicide is challenging because suicide is an extremely personal and sensitive topic [5]. Suicidal behaviour refers to a wide range of suicide-related thoughts (or ideation), cognitions, emotions, and behaviours [6]. It is a term that has been used to categorize behaviour associated with ideas, intentions, motivations, plans, and attempts for suicide. Those who have suicidal thoughts may avoid disclosing these feelings as it triggers feelings of stigmatization.

A recent systematic review [7] supports the use of Cognitive Behavioural Therapy (CBT) and Dialectical Behavioural Therapy (DBT) which are generally effective in reducing the risk of suicidal acts being repeated. However, widespread implementation is limited by the cost of providing face-to-face models of delivery between patients and therapist. The costs in terms of time, money, and potential stigma may also explain why many individuals refuse to seek help [8]. Moreover, CBT relies on imparting psychoeducational information, individuals who do not recognize that they have mental health problems may find this immensely boring. Motivating individuals to engage is another challenge for clinicians aside accessing prevention programs among those who 
live in hard-to-reach locations [9]. Lengthy waiting lists worsen this problem further which identifies the need to explore alternatives with more flexible nature that may remove these constraints. Indeed, advanced digital intervention may be an option as it is both cost-effective and acceptable to the 'hard-to-reach populations'.

\subsection{Implementation of games as digital intervention}

Nowadays $94 \%$ of the young adults own a mobile phone, of which $75 \%$ of users have accessed health information through this device [10]. With the increasing ubiquity of mobile phone ownership in the digitized world, there has been rapid development on the use of digital interventions at a relatively low-cost, convenient and discreet way compared to traditional intervention of seeing a counsellor [11]. With the growth of advanced information technologies, it is argued that digital interventions could be a preferred option to the existing suicide interventions.

Recently, a research team from Harvard University [12] was among the first pioneers to develop a gamified mobile app as a treatment for suicidal thoughts and behaviours (STBs). The app named therapeutic evaluative conditioning (TEC) designed to increase individual's aversion to STBs and decrease aversion to the self. Through simultaneous pairing of targets with emotionally valenced stimuli in this intervention, self-cutting episodes reported by these individuals were significantly reduced, though lasting effects failed to maintain at 1-month follow up. Nevertheless, the research opened up a new direction, that mobile mental health apps can be an increasingly popular platform for self-delivered treatments [13].

Considering that suicidal ideation and suicide risk change rapidly, Mackie and colleagues [14] claimed that the instant access nature of mobile phones may be crucial for those who require immediate assistance. In contrast to the traditional interventions, playing digital games not only exposed players to knowledge acquisition, but provides opportunities in active learning, solving real-life problems, and gaining experience in riskfree surroundings.

\subsection{The underlying causes of suicidal behaviour}

To understand how games could address the underlying causes of suicidal behaviour, it is essential to first explore why many would consider suicide. It seems that cognitive deficits may provide some explanations [15, 16, 17, 18, 19, 20, 21, 22]. Attentional control is a cognitive ability to modulate and control selective attention to relevant information (also referred to as attentional bias) while inhibiting irrelevant information. When suicidal thoughts become overwhelming at a crisis, it is especially important that individuals can resist and inhibit the urge to self-harm. Past research using cognitive tasks revealed how cognitive deficits were associated with suicidal behaviour. For example, the emotional Stroop task is similar to the colour Stroop task whereby participants are instructed to name the colour of words. As emotional and neutral words are used, researchers can assess how emotional distraction interferes with colour-naming of words. 
In an emotional Stroop task ${ }^{1}$ paradigm [23], individuals with attention deficits had more difficulty attending to a target whilst diverting attention away from negative stimuli. This implies that STBs are associated with an attentional bias to particular life events, difficulty in inhibiting irrelevant emotional distractions, and insufficient working memory capacity to solve problems. Consistently, previous studies [15, 24] have explored depressed populations, and found specific attentional bias towards disorder-related cues. Similarly, individuals with STB also exhibited specific bias towards words such as suicide and cutting were more likely to go on to make a suicide attempt. This pattern is particularly useful for clinicians to predict, above and beyond other clinical predictors (e.g., depression, past history of attempt).

Building upon the existing evidence, if cognitive deficits are apparent among suicidal individuals, assessing the extent of such deficits could provide indirect indication on the severity of suicide risk $[15,24]$. With the argument that attentional control is important, recent research [26, 27] showed that attentional biases to emotional information can be trained or untrained among individuals using a cognitive training called Attention Bias Modification (ABM). The design of ABM is based on a dot-probe task. In the task, participants are instructed to focus on a fixation cross at the center of a screen whilst two emotionally salient stimuli (e.g. one angry and one happy face) appear at a random location on either side of the cross. A dot is then be presented at one of the two sides. Participants have to press a button to indicate whether the dot appeared on the left or right. Ferrari et al. [27] assessed the outcome of training through the measures of mood response to stress, depressive symptoms, and cognitive abilities. The results consistently showed that the dot-probe-based ABM training reduced depressive symptoms and attentional bias after two weeks of the intervention. The training was therapeutically effective compared to a placebo (i.e. sham $\mathrm{ABM}$ ) and revealed that the dot-probe task can monitor the effectiveness of other interventions (e.g., CBT and meditation training) and act as an objective measure for cognitive improvements.

The ABM intervention can be administered online and can be a relatively lowcost intervention alongside conventional treatments. Moreover, the ABM intervention was reported to help reduce such bias in depressed patients and therefore have a therapeutic value (see Figure 1. as an example). Currently, the ABM intervention has moved out of the laboratory and is widely available to the public. One example is MindHabits [29] which includes games such as finding a happy stimulus from an array of images. However, the game aims to help students cope with stress, and social rejection issues, but not suicidal behaviour, which reflects the need to develop digital suicide intervention despite the predicted rise in the young adult population. 

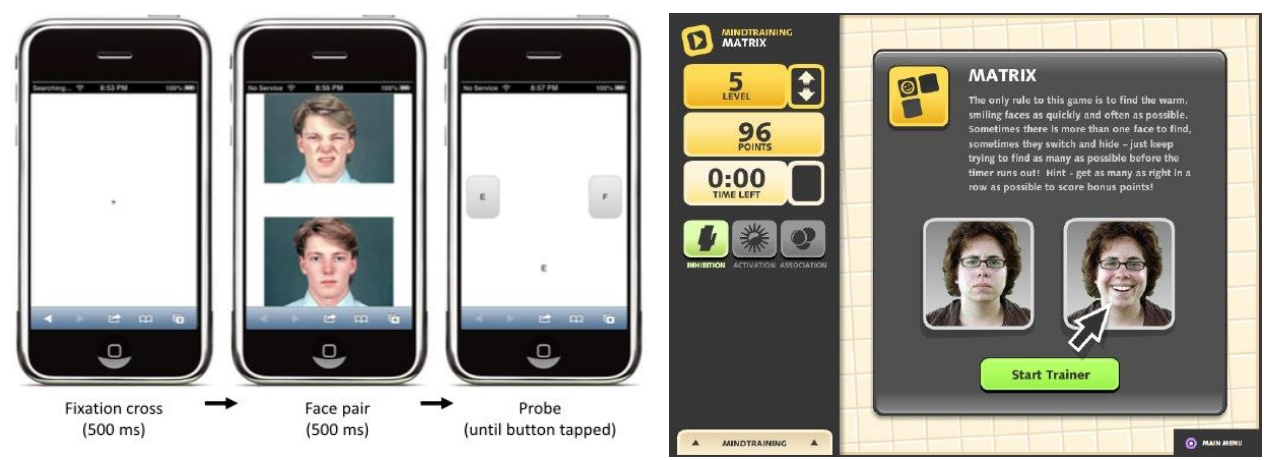

Figure 1. An example of ABM design and MindHabits adapted from ABM design. Adapted with permission from [28] Attention Bias Modification Training Via Smartphone to Reduce Social Anxiety: A Randomized, Controlled Multi-Session Experiment. Cognitive Therapy and Research. Accessed April, 2019.

\subsection{Issues with the existing digital interventions}

Although the majority of digital interventions are effective compared to the treatment as usual group, there are a number of issues that need to be addressed [30]. One problem is the lack of patient adherence. For instance, the lack of lasting effects to maintain at 1month follow up is apparent in many digitized interventions $[12,32]$. Two thirds of users reported only using it for a month, 35\% reported using the digitized intervention for a week or less. This means that although those who used it generally enjoyed it and adherence to the program was high [32], there is still a big challenge in sustaining player's long-term engagement in this intervention. From the researchers' perspectives, their primary goal is to measure the effectiveness of implementing an intervention. In contrast, the game designers often focus on the process of an intervention [31]. Factors to consider are therefore separated out into issues of uptake (i.e. recruitment challenges), attention (i.e. to engage participants in an intervention), and retention (i.e. to maintain individuals to use it). These factors are often less considered by researchers creating discrepancies among them. Game developers that create games without oversight by mental health professionals often risk both ethical issues and conflict with therapeutic goals. They may also not create games most suited for their users due to lack of understanding of users' behaviours. Mandryk and Birk [33] commented that individuals with depression may have difficulties activating their behaviours with motivation. Simple tasks such as getting out of bed can be a major challenge for individuals suffering from STB, with even greater challenge to motivate them to play games. Similarly, with extensive psychological knowledge and practice, researchers may know little about how to design, personalize, or market games for interventions, because they are not familiar with the gaming habits, platform preferences, or genre preferences of these individuals [33].

\section{Conclusion}

The main issue highlighted in this paper is that digitized gamified intervention is a radically new approach to promote mental health, but despite its rapid development in the existing market and an ongoing rise in suicide rates, little attention has been paid to young population with suicidal thoughts and behaviours. Mental health professionals and game 
designers both try hard to develop digital interventions in mental health but the disparity between them creates another barrier to the development of digitized suicide prevention programs. Consequently, the lack of cooperation between game designers and mental health researchers, as well as the exclusion of individuals with lived experience leads to a lack of games that are both beneficial and engaging. Many games have been created as a result with questionable effectiveness and problems with engaging players. Failure to close the research-practice gap indicate that for both researchers and game designers there is still a lot to learn about how to implement these types of interventions in the reality with vulnerable players.

\section{Acknowledgements}

The author consolidated the ideas and wrote the paper. Alexandra Pitman provided comments on the paper and Graham Smith proofread the manuscript.

Ethical approval: Not applicable.

Funding: none

\section{References}

[1] "Demographics of Mobile Device Ownership and Adoption in the United States," Pew Research Center: Internet, Science \& Tech. [Online]. Available: http://www.pewinternet.org/fact-sheet/mobile/. [Accessed: 04-Jan-2020].

[2] "Suicide," National Institute of Mental Health. [Online]. Available: https://www.nimh.nih.gov/health/statistics/suicide.shtml. [Accessed: 04-Jan-2020].

[3] L. Biddle, J. Donovan, D. Sharp, and D. Gunnell, "Explaining non-help-seeking amongst young adults with mental distress: a dynamic interpretive model of illness behaviour," Sociology of Health \& Illness, vol. 29, no. 7, pp. 983-1002, 2007, doi: 10.1111/j.1467-9566.2007.01030.x.

[4] S. Fortune, J. Sinclair, and K. Hawton, "Help-seeking before and after episodes of self-harm: a descriptive study in school pupils in England," BMC Public Health, vol. 8, no. 1, p. 369, 2008/10/24 2008, doi: 10.1186/1471-2458-8-369.

[5] M. K. Nock, F. Ramirez, and O. Rankin, "Advancing Our Understanding of the Who, When, and Why of Suicide Risk," JAMA Psychiatry, vol. 76, no. 1, pp. 11-12, 2019, doi: 10.1001/jamapsychiatry.2018.3164.

[6] E. D. Klonsky, A. M. May, and B. Y. Saffer, "Suicide, Suicide Attempts, and Suicidal Ideation," Annual Review of Clinical Psychology, vol. 12, no. 1, pp. 307-330, 2016/03/28 2016, doi: 10.1146/annurevclinpsy-021815-093204.

[7] K. Hawton, K. G. Witt, T. L. T. Salisbury, E. Arensman, D. Gunnell, P. Hazell, E. Townsend, and K. V. Heeringen, "Psychosocial interventions for self-harm in adults," Cochrane Database of Systematic Reviews, Dec. 2016. Issue 5. Art. No.: CD012189. DOI: 10.1002/14651858.CD012189

[8] H. Scholten, M. Malmberg, A. Lobel, C. Engels, and I. Granic, "A Randomized Controlled Trial to Test the Effectiveness of an Immersive 3D Video Game for Anxiety Prevention among Adolescents: e0147763," PLoS ONE, vol. 11, no. 1, 2016, doi: 10.1371/journal.pone.0147763.

[9] E. Goldberg, "Rural areas have the highest Suicide Rates and fewest Mental Health workers," HuffPost, 18-Jun-2018. [Online]. Available: https://www.huffpost.com/entry/rural-suicide-rates-mentalhealth_n_5b22dd28e4b0d4fc01fcc098. [Accessed: 04-Jan-2020].

[10] "Demographics of Mobile Device Ownership and Adoption in the United States," Pew Research Center: Internet, Science \& Tech. [Online]. Available: http://www.pewinternet.org/fact-sheet/mobile/. [Accessed: 04-Jan-2020].

[11] M. E. Larsen, J. Nicholas, and H. Christensen, "A Systematic Assessment of Smartphone Tools for Suicide Prevention. (Report)," PLoS ONE, vol. 11, no. 4, 2016, doi: 10.1371/journal.pone.0152285.

[12] J. C. Franklin, K. R. Fox, C. R. Franklin, E. M. Kleiman, J. D. Ribeiro, A. C. Jaroszewski, J. M. Hooley, and M. K. Nock, "A brief mobile app reduces nonsuicidal and suicidal self-injury: Evidence from three randomized controlled trials.," Journal of Consulting and Clinical Psychology, vol. 84, no. 6, pp. 544557, 2016, doi: 10.1037/ccp0000093.

[13] A. Emily, "Mental health: There's an app for that," Nature, vol. 532, no. 7597, p. 20, 2016, doi: $10.1038 / 532020 \mathrm{a}$. 
[14] C. Mackie, N. Dunn, S. Maclean, V. Testa, M. Heisel, and S. Hatcher, "A qualitative study of a blended therapy using problem solving therapy with a customised smartphone app in men who present to hospital with intentional self-harm," Evidence Based Mental Health, vol. 20, no. 4, pp. 118-122, 2017 [Online]. Available: http://ebmh.bmj.com/content/20/4/118.full.pdf

[15] C. Cha, S. Najmi, J. Park, C. Finn, and M. K. Nock, "Attentional bias toward suicide-related stimuli predicts suicidal behavior," Journal of Abnormal Psychology, vol. 119, no. 3, 2010, doi: 10.1037/a0019710.

[16] F. Jollant, N. S. Lawrence, V. Giampietro, M. J. Brammer, M. A. Fullana, D. Drapier, P. Courtet, and M. L. Phillips, "Orbitofrontal Cortex Response to Angry Faces in Men with Histories of Suicide Attempts," American Journal of Psychiatry, vol. 165, no. 6, pp. 740-748, 2008, doi: 10.1176/appi.ajp.2008.07081239.

[17] F. Jollant, N. S. Lawrence, E. Olie, O. Odaly, A. Malafosse, P. Courtet, and M. L. Phillips, "Decreased activation of lateral orbitofrontal cortex during risky choices under uncertainty is associated with disadvantageous decision-making and suicidal behavior," NeuroImage, vol. 51, no. 3, pp. 1275-1281, 2010, doi: 10.1016/j.neuroimage.2010.03.027.

[18] J. G. Keilp, M. Gorlyn, M. A. Oquendo, A. K. Burke, and J. J. Mann, "Attention deficit in depressed suicide attempters," Psychiatry Research, vol. 159, no. 1-2, pp. 7-17, 2008, doi: 10.1016/j.psychres.2007.08.020.

[19] J. G. Keilp, M. Gorlyn, M. Russell, M. A. Oquendo, A. K. Burke, J. Harkavy-Friedman, and J. J. Mann, "Neuropsychological function and suicidal behavior: attention control, memory and executive dysfunction in suicide attempt," Psychological Medicine, vol. 43, no. 3, pp. 539-551, Oct. 2013, doi: 10.1017/S0033291712001419.

[20] J. J. Mann, D. Currier, B. Stanley, M. A. Oquendo, L. V. Amsel, and S. P. Ellis, "Can biological tests assist prediction of suicide in mood disorders?" Int. J. Neuropsychopharm., vol. 9, no. 4, pp. 465-474, 2006, doi: 10.1017/S1461145705005687.

[21] E. Ong and C. Thompson, "The Importance of Coping and Emotion Regulation in the Occurrence of Suicidal Behavior," Psychological Reports, vol. 122, no. 4, pp. 1192-1210, 2019, doi: 10.1177/0033294118781855.

[22] J. Westheide, B. B. Quednow, K.-U. Kuhn, C. Hoppe, D. Cooper-Mahkorn, B. Hawellek, P. Eichler, W. Maier, and M. Wagner, "Executive performance of depressed suicide attempters: the role of suicidal ideation," European Archives of Psychiatry and Clinical Neuroscience, vol. 258, no. 7, pp. 414-421, Nov. 2008, doi: 10.1007/s00406-008-0811-1.

[23] C. Thompson and E. L. C. Ong, "The Association Between Suicidal Behavior, Attentional Control, and Frontal Asymmetry," Frontiers in Psychiatry, vol. 9, 2018, doi: 10.3389/fpsyt.2018.00079.

[24] Y. Chung and E. L. Jeglic, "Detecting Suicide Risk Among College Students: A Test of the Predictive Validity of the Modified Emotional Stroop Task," Suicide and Life-Threatening Behavior, vol. 47, no. 4, pp. 398-409, 2017, doi: 10.1111/sltb.12287.

[25] M. M. Bradley, and P. J. Lang, "Affective norms for English words (ANEW): Stimuli, instruction manual and affective ratings," Technical report C-1. Gainesvill, FL: The Center for Research in Psychophysiology, University of Florida 1999, Available: https://www.uvm.edu/pdodds/teaching/courses/2009-08UVM300/docs/others/everything/bradley1999a.pdf [Accessed: 04-Jan-2020].

[26] M. Boffo, R. Willemen, T. Pronk, R. W. Wiers, and G. Dom, "Effectiveness of two web-based cognitive bias modification interventions targeting approach and attentional bias in gambling problems: study protocol for a pilot randomised controlled trial," Trials, vol. 18, no. 1, Mar. 2017, doi: 10.1186/s13063017-2190-2.

[27] G. R. A. Ferrari, E. S. Becker, F. Smit, M. Rinck, and J. Spijker, "Investigating the (cost-) effectiveness of attention bias modification (ABM) for outpatients with major depressive disorder (MDD): a randomized controlled trial protocol," BMC Psychiatry, vol. 16, no. 1, Mar. 2016, doi: 10.1186/s12888016-1085-1.

[28] P. M. Enock, S. G. Hofmann, and R. J. Mcnally, "Attention Bias Modification Training Via Smartphone to Reduce Social Anxiety: A Randomized, Controlled Multi-Session Experiment," Cognitive Therapy and Research, vol. 38, no. 2, pp. 200-216, Apr. 2014, doi: 10.1007/s10608-014-9606-z.

[29] "Stress Relief Game," MindHabits. [Online]. Available: https://www.mindhabits.com/press.php. [Accessed: 04-Jan-2020].

[30] M. V. Birk and R. L. Mandryk, "Improving the Efficacy of Cognitive Training for Digital Mental Health Interventions Through Avatar Customization: Crowdsourced Quasi-Experimental Study," Journal of Medical Internet Research, vol. 21, no. 1, Aug. 2019, doi: 10.2196/10133.

[31] I. Miralles and C. Granell, "Considerations for Designing Context-Aware Mobile Apps for Mental Health Interventions," International Journal of Environmental Research and Public Health, vol. 16, no. 7, p. 1197, Mar. 2019, doi: 10.3390/ijerph16071197.

[32] Evaluation of $\quad$ SPARX. (2016). Retrieved from https://www.health.govt.nz/system/files/documents/publications/evaluation-sparx-dec16.pdf

[33] R. L. Mandryk and M. V. Birk, "Toward Game-Based Digital Mental Health Interventions: Player Habits and Preferences,” Journal of Medical Internet Research, vol. 19, no. 4, 2017, doi: 10.2196/jmir.6906. 